\title{
REGULATION CONTROL OF AN UNDERACTUATED MECHANICAL SYSTEM WITH DISCONTINUOUS FRICTION AND BACKLASH
}

\author{
RAÚl RASCÓN $^{a, *}$, DAVID ROSAS $^{a}$, DANIEL HERNANDEZ-BALBUENA ${ }^{a}$ \\ ${ }^{a}$ Faculty of Engineering, UABC Autonomous University of Baja California \\ Blvd. Benito Juárez y Calle de la Normal S/N, 21280, Mexicali, Baja California, México \\ e-mail: \{raul.rascon, drosas, dhernan\} @uabc.edu.mx
}

\begin{abstract}
In this work, the problem of position regulation control is addressed for a 2DOF underactuated mechanical system with friction and backlash. For this purpose, a method combining sliding mode and $H_{\infty}$ control is developed. We prove that the application of the method to the nonlinear model considered results in an asymptotically stable equilibria set. Moreover, it is possible to achieve a sufficiently small and bounded steady-state position error even in the presence of disturbances by employing the proposed technique. That is, the developed controller is able to account not only for unmatched external perturbations and model discrepancies of the test rig considered, but also for matched bounded perturbations. The control methodology is presented from both the theoretical and experimental angles to demonstrate the good performance of the proposed controller.
\end{abstract}

Keywords: underactuated mechanical system, sliding mode control, $H_{\infty}$ control, discontinuous observer.

\section{Introduction}

1.1. Motivation and methodology. A major problem in control engineering is robust feedback design that asymptotically stabilizes a nominal mechanical system while also attenuating the influence of parameter variations and external disturbances. In mechanical systems this control objective becomes difficult when the system motion is constrained by phenomena like backlash. In the last decade, this problem has been intensely studied and research efforts have been made to control systems with different types of constraints (Mansard and Khatib, 2008; Potini et al., 2006; Perez et al., 2010). Nonetheless, parameter variations and disturbances are not frequently considered. Some important references fundamental in this respect are given by Brogliato (1999) as well as Leine and Van de Wouw (2010).

A methodology widely spread due to its effectiveness against matched bounded disturbances and perturbations is sliding mode control (see Utkin, 1992). The main feature of this class of controllers is to allow sliding mode to occur on a prescribed switching surface, so that the system is governed by the sliding equation

* Corresponding author only, and remains insensitive to a class of disturbances and parameter variations (Utkin, 1978). This control method has been successfully tested for motion control of robotic manipulators (see Sabanovic et al., 2008) and the references therein, and also, previous work on sliding modes control in constrained robots has been conducted by Lian and Lin (1998). Durmaz et al. (2011) proposed sliding mode control design based upon adaptive sliding surfaces moving with varying slopes and offsets for a class of nonlinear systems, and demonstrated the effectiveness of the proposed approach by means of a simulated maneuver of the aircraft.

Moreover, $H_{\infty}$ control methodology has been shown to be effective in the control of systems affected by unknown disturbances, and also in systems where only incomplete and imperfect state measurements are available; these can include unmatched uncertainties and disturbances. Surveys of the methods for the $H_{\infty}$ case are given by Doyle et al. (1989) and Isidori (2000).

Due to the particular advantages of both control methodologies, it is of interest to design a control technique to combine the robustness properties of sliding mode and $H_{\infty}$ control, in order to design a controller which is capable to handle the above mentioned factors, such as backlash, Coulomb friction, 
matched and unmatched perturbations, thereby yielding good performance on real systems. In recent research works, the combination of sliding mode control with $H_{\infty}$ methodology has shown effectiveness in controlling switched systems (Castaños and Fridman, 2011; Lian and Zhao, 2010; Ghafari-Kashani et al., 2010). Brahim et al. (2015) presented an $H_{\infty}$ sliding mode observer for nonlinear systems with simultaneous actuator and sensor faults.

The problem addressed in the present paper is position regulation through output feedback in a mechanical system with backlash, where the latter is considered a clearance between mechanical parts. Some applications include machine tool tables, where backlash is undesirable in precision positioning. These types of systems can constraint the free motion of the actuated link due to the effect of the clearance. In such systems, collisions between parts may occur. Some previous work on mechanical systems with position constraints, such as backlash, includes that by Mansard and Khatib (2008) or Menini and Tornambe (2001). Moreover, an analysis of observability and controllability of sandwich systems with backlash is presented by Luo et al. (2015).

Some efforts have been made to control constrained mechanical systems using a wide range of methodologies such as sliding mode control (Sabanovic et al., 2008), predictive control (Adetola et al., 2009), and optimal control (Christophersen, 2007), among others. The literature on the control of perturbed and uncertain mechanical systems under constraints is scarce. For example, the problem of unilateral constraints on the position is considered by Brogliato et al. (1997). Tseng (2005) set fourth a PID-type controller for constrained robots added to a mixed $H_{2} / H_{\infty}$ control to attenuate the influence of disturbances and uncertainties. Chiu et al. (2004) designed a robust motion/force tracking control for constrained uncertain robots. On the other hand, Chang and Lee (1999) proposed a dynamic position feedback/force control designed for constrained robots actuated by DC motors.

In previous works, Rascón et al. (2012) addressed a position regulation problem in an underactuated mechanical system with a clearance between parts, where a Dahl friction type was considered in both links; a numerical comparison was made using the proposed sliding mode- $H_{\infty}$ controller and a first-order sliding mode controller. Recently, Rascón et al. (2016) considered the implementation and control design of a class of Lagrangian systems, particularly underactuated mechanical systems with friction and backlash; the control methodology used for this purpose was $H_{\infty}$, while an experimental comparison was carried out using the proposed controller and a PI algorithm.
1.2. Strategy and contribution. In this study, a method of control design for a mechanical system with backlash is proposed, where viscous friction and dry friction of Coulomb type are taken into account. Moreover, it is assumed that the system can be affected by external perturbations, both matched and unmatched. Only position measurements are available to be used as feedback; because of this, a discontinuous velocity observer is proposed. The proposed control law is based on integral sliding mode and $H_{\infty}$ control, and also an exponential reaching law is used in order to reduce the convergence time to the sliding surface. A previous work involving an integral/exponential gain law to absorb matched perturbations is that by Zhu and Khayati (2015). In contrast to the $H_{\infty}$ controller used by Rascón et al. (2016), in this work the authors proposed a sliding mode controller which includes an $H_{\infty}$ control stage in the sliding surface; this controller is capable of handling matched and unmatched perturbations. The former could be eliminated if they are bounded, and the latter are mitigated through the $H_{\infty}$ control stage. Another advantage of the present approach is the use of a finite time observer which renders better closed-loop performance of the system.

The closed-loop stability of the mechanical system is proved using quadratic functions; some references can be found in the works of Paden and Sastry (1987), Shevitz and Paden (1994), Kazerooni (1990) or Branicky (1998). Through the usage of quadratic functions we can ensure finite time convergence of the trajectories to the sliding surface.

Preliminary work using a sliding mode and $H_{\infty}$ controller is done by Rascón et al. (2012; 2014), who do not consider Coulomb friction. There a differentiable approximation of the dead zone model of backlash was also used moreover, the velocity observer and the exponential reaching law were not presented. In order to validate the theoretical analysis, an experimental comparison is made using the proposed controller against a PI algorithm.

1.3. Paper structure. The paper is outlined as follows. In Section 2, we describe the mechanical system with the dead-zone model of backlash; there is also a transformation of the mechanical model based on the position error made for control purposes. In Section 3, output feedback design is presented, corresponding to sliding mode control design and its convergence analysis to the sliding surface. In Section 4, the synthesis of the $H_{\infty}$ control stage is addressed. Later, in Section 5 there is presented the development of a finite time velocity observer and its stability proof. Section 6 discusses experimental results performed in an underactuated mechanical system with backlash. Finally, Section 7 presents some conclusions. 


\section{Problem statement}

The main concern of this work is regulation control design (and its stability analysis) of a mechanical system subject to backlash and Coulomb friction (see Fig. 10. This is a basic system, described by a rather simple model. However, it is similar to those presented in many mechanical systems, especially the ones with clearances. It can display an important dynamical behavior like rebounds due to collisions with the constraint, which may risk the integrity of a mechanical device. The system consists of two masses subject to viscous friction, which are linked by a spring with backlash. Hence, we design the controller to reduce the presence of the backlash phenomenon besides having good regulation. The equations of motion of the open-loop mechanical

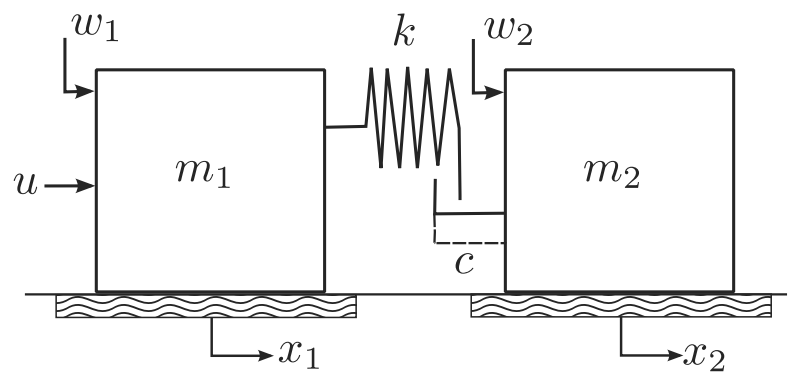

Fig. 1. Constrained mechanical system.

system can be expressed in joint coordinates as

$$
\begin{aligned}
m_{1} \ddot{x}_{1}+F_{1}\left(\dot{x}_{1}\right)+f\left(x_{1}, x_{2}\right) & =u+w_{1}(t), \\
m_{2} \ddot{x}_{2}+F_{2}\left(\dot{x}_{2}\right) & =f\left(x_{1}, x_{2}\right)+w_{2}(t),
\end{aligned}
$$

where $m_{i}, x_{i}(t), \dot{x}_{i}(t), \ddot{x}_{i}(t), i=1,2$, represent the mass, displacement, velocity and acceleration of the $i$-th link, respectively, and the parameters $F_{i}\left(\dot{x}_{i}\right)$ are the viscous friction plus Coulomb friction. Moreover, $u$ stands for the control force and $f\left(x_{1}, x_{2}\right)$ is the contact force between masses caused by the backlash. The perturbation $w_{1}(t) \in \mathbb{R}$ constitutes a matched perturbation, i.e., this is, a perturbation that comes into the state space equations as well as the control input (for example, a completely actuated link). In contrast, $w_{2}(t) \in \mathbb{R}$ is an unmatched perturbation, which affects the unactuated link.

The transmitted contact force $f\left(x_{1}, x_{2}\right)$ between the masses is given by

$$
f\left(x_{1}, x_{2}\right)= \begin{cases}k\left(\delta x-\frac{c}{2}\right) & \text { if } \delta x \geq \frac{c}{2} \\ 0 & \text { if }-\frac{c}{2}<\delta x<\frac{c}{2}, \\ k\left(\delta x+\frac{c}{2}\right) & \text { if } \delta x \leq-\frac{c}{2}\end{cases}
$$

where $\delta x=x_{1}-x_{2}, k$ is the spring stiffness and $c$ is the size of the backlash. We assume that the mass density is homogeneous.

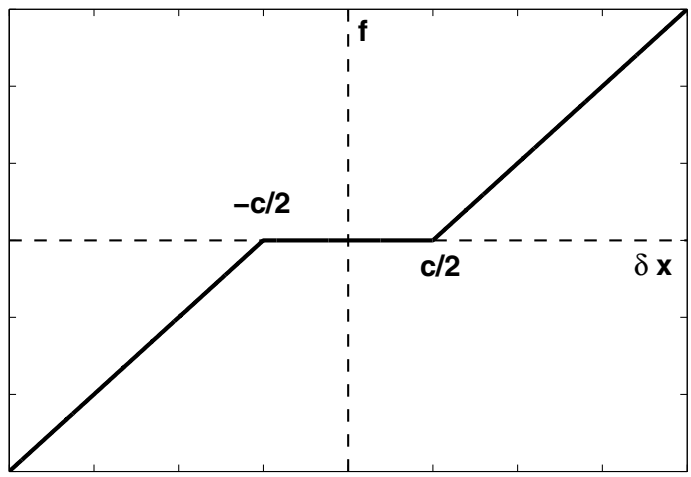

Fig. 2. Dead-zone model of backlash.

The expression (2) can be rewritten as

$$
f\left(x_{1}, x_{2}\right)=\frac{k}{2}\left(2 \delta x+\left|\delta x-\frac{c}{2}\right|-\left|\delta x+\frac{c}{2}\right|\right) .
$$

The behavior of the dead-zone model of backlash can be seen in Fig. 2. The friction model chosen for the treatment is the static Coulomb model augmented with viscous friction:

$$
F_{j}=\sigma_{0 j} \dot{x}_{j}+F_{c j} \operatorname{sign}\left(\dot{x}_{j}\right), \quad j=1,2,
$$

where $\sigma_{0 j}>0$ and $F_{c j}>0$ are the viscous friction coefficient and the Coulomb friction level, respectively, corresponding to the $j$-th manipulator joint. Moreover, the sign is the signum function, defined by

$$
\operatorname{sign}(\dot{x})= \begin{cases}1 & \text { if } \dot{x}>0, \\ 0 & \text { if } \dot{x}=0, \\ -1 & \text { if } \dot{x}<0 .\end{cases}
$$

Since the right-hand side of Eqn. (1) has discontinuous terms due to Coulomb friction, the solutions of the system (1) are understood in the Filippov sense and according to the simplest convex definition given by Filippov (1988, Chapter 2).

Provided that mass position $x_{1}(t) \in \mathbb{R}$ and mass position $x_{2}(t) \in \mathbb{R}$ are the only available measurements of the system, the above open-loop model (1), (4) possesses a multivalued set of equilibria $\left(\bar{x}_{1}, \bar{x}_{2}\right)$, with $\bar{x}_{1}=\zeta$, where $\zeta$ is any constant and $\bar{x}_{2} \in\left[\zeta-c / 2-F_{c 2} / k, \zeta+c / 2+\right.$ $\left.F_{c 2} / k\right]$.

According to the proposal of Aguilar et al. (2003), some nonlinear expressions, such as (3), can be considered in $H_{\infty}$ controller design for the system (1), whose only measurable outputs $y(t) \in \mathbb{R}^{2}$ are the positions of the masses, that is

$$
y=\left[\begin{array}{l}
x_{1} \\
x_{2}
\end{array}\right] .
$$


The objective of the sliding mode $H_{\infty}$ controller is to regulate the position of the mass $x_{2}$ towards a reference $x_{2 d} \in \mathbb{R}$, which is $\lim _{t \rightarrow \infty}\left\|x_{2}(t)-x_{2_{d}}\right\|<\epsilon, \epsilon$ being a constant sufficiently small in spite of the presence of bounded matched and bounded unmatched perturbations, and the presence of Coulomb friction in both masses.

The role of the sliding mode stage is to eliminate the effect of $w_{1}$ and $F_{c 1} \operatorname{sign}\left(\dot{x}_{1}\right)$, and the purpose of the $H_{\infty}$ control stage is to attenuate the unmatched perturbation $w_{2}$, and $F_{c 2} \operatorname{sign}\left(\dot{x}_{2}\right)$. In fact, it is important to mention that inadequate control design to eliminate the matched perturbations can even amplify the unmatched perturbations (Castaños and Fridman, 2006). Given the nonlinear underactuated control system (1) with the contact force (3), a sliding mode control will be designed in such a way that the closed-loop trajectories remain bounded, and the output $x_{2}$ asymptotically decreases to a set around the desired position $x_{2 d}$ via $x_{1 d}$, in the presence of bounded perturbations which satisfy

$$
\sup _{t}\left|w_{1}(t)\right| \leq M, \quad M>0 .
$$

The purpose of $H_{\infty}$ control is to attenuate the influence of external perturbations $w_{2}$ and discontinuous friction $F_{c 2} \operatorname{sign}\left(\dot{x}_{2}\right)$.

Let us consider the following change of coordinates:

$$
\begin{array}{ll}
q_{1}=x_{1}-x_{1_{d}}, & q_{2}=\dot{x}_{1}, \\
q_{3}=x_{2}-x_{2_{d}}, & q_{4}=\dot{x}_{2},
\end{array}
$$

where $x_{2 d}$ is given by

$$
x_{2 d}= \begin{cases}x_{1 d}-\frac{c}{2} & \text { if } x_{1 d}>\frac{c}{2}, \\ 0 & \text { if }\left|x_{1 d}\right| \leq \frac{c}{2} \\ x_{1 d}+\frac{c}{2} & \text { if } x_{1 d}<-\frac{c}{2} .\end{cases}
$$

The expression 9) can be rewritten as

$$
x_{2 d}=\frac{1}{2}\left(2 x_{1 d}+\left|x_{1 d}-\frac{c}{2}\right|-\left|x_{1 d}+\frac{c}{2}\right|\right) .
$$

Therefore, the state space representation of the system (1) according to the change of coordinates in 8 is

$$
\begin{aligned}
& \dot{q}_{1}=q_{2}, \\
& \dot{q}_{2}=\frac{1}{m_{1}}\left[-F_{1}\left(q_{2}\right)-f\left(q_{1}+x_{1_{d}}, q_{3}+x_{2_{d}}\right)+u+w_{1}\right], \\
& \dot{q}_{3}=q_{4} \\
& \dot{q}_{4}=\frac{1}{m_{2}}\left[-F_{2}\left(q_{4}\right)+f\left(q_{1}+x_{1_{d}}, q_{3}+x_{2_{d}}\right)+w_{2}\right],
\end{aligned}
$$

where

$$
\begin{array}{r}
f\left(q_{1}+x_{1_{d}}, q_{3}+x_{2_{d}}\right) \\
=\frac{k}{2}\left(2 \delta q+\left|\delta q-\frac{c}{2}\right|-\left|\delta q+\frac{c}{2}\right|\right), \\
\text { and } \delta q=q_{1}-q_{3}-\frac{1}{2}\left|x_{1 d}-\frac{c}{2}\right|+\frac{1}{2}\left|x_{1 d}+\frac{c}{2}\right| .
\end{array}
$$

\section{Design procedure}

The control objective consists in stabilizing the position of mass $x_{2}(t)$ to a desired reference $x_{2 d}$, that is,

$$
\lim _{t \rightarrow \infty}\left\|q_{3}(t)\right\|=\lim _{t \rightarrow \infty}\left\|x_{2}(t)-x_{2_{d}}\right\|<\epsilon .
$$

This is achieved through closed-loop controller design, specifically using the sliding mode control technique plus an $H_{\infty}$ attenuator. The $H_{\infty}$ stage is incorporated into sliding surface design. The sliding mode control technique is able to eliminate matched perturbations affecting the mechanical system.

3.1. Sliding mode control. Consider the following sliding surface:

$$
s=q_{1}+q_{2}+\int_{0}^{T} q_{1}(t) \mathrm{d} t-\int_{0}^{T} u_{\infty}(t) \mathrm{d} t,
$$

where $u_{\infty}(t) \in \mathbb{R}$ is the $H_{\infty}$ control stage, whose design will be explained in Section 4 through the use of the procedure shown by Aguilar et al. (2003) or Isidori and Astolfi (1992). The aim of designing the sliding surface shown in (14) will be meaningful in the development of the proof of closed-loop stability. The control law which ensures that the trajectories reach the sliding surface is given by

$$
\begin{aligned}
u= & \sigma_{01} q_{2}+f\left(q_{1}+x_{1_{d}}, q_{3}+x_{2_{d}}\right) \\
& +m_{1}\left[-q_{1}-q_{2}+u_{\infty}-\lambda s-\frac{\beta}{N(s)} \operatorname{sign}(s)\right],
\end{aligned}
$$

where $\lambda$ and $\beta$ are positive constant gains which are tuned to ensure that the movement of the trajectories goes toward the sliding surface. $N(s)$ is the so-called exponential reaching law given by Fallaha et al. (2011), and expressed by

$$
N(s)=\delta_{0}+\left(1-\delta_{0}\right) e^{-\alpha|s|^{p}},
$$

where the constants $0<\delta_{0}<1, p>0$ and $\alpha>0$. Since $N(s)$ is always strictly positive, the exponential reaching law given by (16) does not affect the stability of the reduced order system, as it is proved in the next section.

Substituting the control input (14)-15 into the dynamic system (11), it can be seen that the closed-loop dynamics are given by

$$
\begin{aligned}
& \dot{q}_{1}=q_{2}, \\
& \dot{q}_{2}=-q_{1}-q_{2}-\lambda s-\frac{\beta}{N(s)} \operatorname{sign}(s)+u_{\infty}
\end{aligned}
$$




$$
\begin{aligned}
& -\frac{F_{c 1} \operatorname{sign}\left(q_{2}\right)}{m_{1}}+\frac{w_{1}}{m_{1}}, \\
\dot{q}_{3}= & q_{4}, \\
\dot{q}_{4}= & \frac{1}{m_{2}}\left(-F_{2}\left(q_{4}\right)+f\left(q_{1}+x_{1_{d}}, q_{3}+x_{2_{d}}\right)+w_{2}\right), \\
\dot{s}= & -\lambda s-\frac{\beta}{N(s)} \operatorname{sign}(s)+\frac{w_{1}}{m_{1}} .
\end{aligned}
$$

In one of the next sections we propose a finite-time observer in order to estimate the velocity vector, which is needed to implement the control input (14)-(15).

\subsection{Analysis of the existence of sliding modes.} First of all, let us analyze the stability properties of the closed-loop system (17). The convergence of the output variables $q_{1}$ and $q_{3}$ will be analyzed as follows: firstly we will prove that the trajectories of (11) are attracted by the sliding surface (14) and converge to it in finite time; then, once the trajectories are on the sliding surface, it will be proved that $\left(q_{1}, q_{2}\right)$ decreases exponentially to $(0,0)$; finally, it will be proved that trajectories $\left(q_{3}, q_{4}\right)$ converge asymptotically to a constant value $(\epsilon, 0)$.

According to Utkin (1978), the existence of sliding modes can be ensured if $s \dot{s}<0$ is satisfied. The expression of $s \dot{s}$ can be developed as follows:

$$
\begin{aligned}
s \dot{s} & =s\left(-\lambda s-\frac{\beta}{N(s)} \operatorname{sign}(s)-\frac{F_{c 1} \operatorname{sign}\left(q_{2}\right)}{m_{1}}+\frac{w_{1}}{m_{1}}\right) \\
& \leq-\lambda s^{2}-\left(\frac{\beta}{N(s)}-\frac{F_{c 1}}{m_{1}}-\frac{M}{m_{1}}\right)|s|,
\end{aligned}
$$

from which the existence of sliding modes on $s=$ 0 can be determined, while the condition $\beta / N(s)-$ $F_{c 1} / m_{1}-M / m_{1}>0$ remains valid. Moreover, from the exponential sliding surface $N(s)=\delta_{0}+\left(1-\delta_{0}\right) e^{-\alpha|s|^{p}}$, one can see the following limit cases:

$$
\lim _{s \rightarrow 0} N(s)=1, \quad \lim _{s \rightarrow \infty} N(s)=\delta_{0} .
$$

Therefore $\beta / N(s) \in\left[\beta, \beta / \delta_{0}\right]$. This means that $\beta / N(s)$ increases as $|s|$ increases and, consequently, the convergence rate to $s=0$ will be faster. The expression for the convergence rate $t_{f}$ is shown in what follows. On the other hand, if $|s|$ decreases, then $N(s)$ approaches 1 , and $\beta / N(s)$ converges to $\beta$. This means that, when the system's trajectories approach the reference, $\beta / N(s)$ gradually decreases in order to reduce the control effort. Thus, the exponential reaching law allows the controller to dynamically adapt to variations in $s$.

If $\delta_{0}$ is chosen to be equal to 1 , the reaching law (18) becomes identical to the conventional reaching law $\dot{s}=-\lambda s-\beta \cdot \operatorname{sign}(s)+\gamma(t, q)$, where $\gamma(t, q)$ includes the perturbations and Coulomb friction. Therefore, the conventional reaching law is a subset of the present approach.

Also, we can demonstrate finite time convergence of the trajectories to the surface $s=0$ using the quadratic function

$$
V(s)=s^{2},
$$

and compute its time derivative along the solutions of (17),

$$
\begin{aligned}
\dot{V} & \leq-2 \lambda s^{2}-2\left(\frac{\beta}{N(s)}-\frac{F_{c 1}}{m_{1}}-\frac{M}{m_{1}}\right)|s| \\
& \leq-2\left(\frac{\beta}{N(s)}-\frac{F_{c 1}}{m_{1}}-\frac{M}{m_{1}}\right)|s| \\
& =-2\left(\frac{\beta}{N(s)}-\frac{F_{c 1}}{m_{1}}-\frac{M}{m_{1}}\right) \sqrt{V},
\end{aligned}
$$

under the initial condition $V\left(t_{0}\right)=V_{0}$. Assuming that $\beta / N(s)>F_{c 1} / m_{1}+M / m_{1}$, we can guarantee the existence of a time $t_{f}$ such that, for all $t>t_{f}$, the function $V$ tends to zero. This can be computed directly from (21), where

$$
V(t)=0 \text { for } t \geq t_{0}+\frac{\sqrt{V_{0}}}{\frac{\beta}{N(s)}-\frac{F_{c 1}+M}{m_{1}}} \triangleq t_{f}
$$

Hence, $V(t)$ converges to zero in finite time and, in consequence, a motion along the manifold $s=0$ occurs in the discontinuous system (17) from $t_{f}$ at most.

Thus, in the sequel, we will assume that the system (17) is in sliding mode, so $s=\dot{s}=0$ for $t \geq t_{f}$. Under these conditions, the dynamics of the system (17) can be reduced to

$$
\begin{aligned}
& \dot{q}_{1}=q_{2} \\
& \dot{q}_{2}=-q_{1}-q_{2}+u_{\infty} \\
& \dot{q}_{3}=q_{4} \\
& \dot{q}_{4}=\frac{1}{m_{2}}\left(-F_{2}\left(q_{4}\right)+f\left(q_{1}+x_{1_{d}}, q_{3}+x_{2_{d}}\right)+w_{2}\right) .
\end{aligned}
$$

Notice that $\dot{q}_{2}$ was obtained from $\dot{s}=0$; this is when the trajectories are on the sliding surface and their dynamics are insensitive to the influence of Coulomb friction and external perturbations. Therefore, the sliding surface $s$ in (14) was designed in accordance with the control objective and the desired reduced order dynamics in 23 .

3.3. Stability analysis. Now, consider $u_{\infty}=0$ and $w_{2}(t)=F_{c 2}=0$. It is possible to show that, when $u_{\infty}=0, q \in \mathbb{R}^{4}$ converge to an equilibrium point $\bar{q}=(0,0, \epsilon, 0)$, with $|\epsilon| \leq c / 2$. To this end, notice from (23) that variables $q_{1}$ and $q_{2}$ are decoupled from variables $q_{3}$ and $q_{4}$. If we consider the subsystem

$$
\begin{aligned}
& \dot{q}_{1}=q_{2}, \\
& \dot{q}_{2}=-q_{1}-q_{2},
\end{aligned}
$$


according to Khalil (2002, Definition 4.5), it can be concluded that $q_{1}$ and $q_{2}$ exponentially decay to zero.

Considering the stationary case, the residual dynamics are

$$
\begin{aligned}
& \dot{q}_{3}=q_{4} \\
& \dot{q}_{4}=\frac{1}{m_{2}}\left[-\sigma_{02} q_{4}+f\left(q_{1}+x_{1_{d}}, q_{3}+x_{2_{d}}\right)\right],
\end{aligned}
$$

where $f\left(q_{1}+x_{1_{d}}, q_{3}+x_{2_{d}}\right)$ is given in (12), in accordance with what is shown in (3) and Fig. 2. Given that $q_{1}$ converges exponentially to zero, the dynamics of system (24) can be analyzed from

$$
\begin{aligned}
\dot{q}_{3}= & q_{4}, \\
\dot{q}_{4}= & -\frac{\sigma_{02}}{m_{2}} q_{4}-\frac{k}{m_{2}} q_{3}+\frac{k}{2 m_{2}}\left(\left|\delta q-\frac{c}{2}\right|\right. \\
& \left.+\left|x_{1 d}+\frac{c}{2}\right|-\left|\delta q+\frac{c}{2}\right|-\left|x_{1 d}-\frac{c}{2}\right|\right),
\end{aligned}
$$

where now

$$
\left|\delta q-\frac{c}{2}\right|=\left|-q_{3}-\frac{c}{2}-\frac{1}{2}\right| x_{1 d}-\frac{c}{2}\left|+\frac{1}{2}\right| x_{1 d}+\frac{c}{2}||
$$

and

$$
\left|\delta q+\frac{c}{2}\right|=\left|-q_{3}+\frac{c}{2}-\frac{1}{2}\right| x_{1 d}-\frac{c}{2}\left|+\frac{1}{2}\right| x_{1 d}+\frac{c}{2}|| .
$$

Let us consider the differentiable and nonnegative function $V: \mathbb{R}^{2} \rightarrow \mathbb{R}$,

$$
\begin{aligned}
V\left(q_{3}, q_{4}\right)= & \frac{1}{2} q_{4}^{2} \\
& +\frac{k}{2 m_{2}} \begin{cases}q_{3}^{2}+c q_{3}+\frac{c^{2}}{4} & \text { if } q_{3}<-\frac{c}{2}, \\
0 & \text { if }\left|q_{3}\right| \leq \frac{c}{2} \\
q_{3}^{2}-c q_{3}+\frac{c^{2}}{4} & \text { if } q_{3}>\frac{c}{2}\end{cases}
\end{aligned}
$$

Using the aforementioned function $V\left(q_{3}, q_{4}\right)$, we have for the three cases that

$$
\dot{V}\left(q_{3}, q_{4}\right)=-\frac{\sigma_{02}}{m_{2}} q_{4}^{2} \leq 0,
$$

which is a negative semi-definite function. Now, given that $V\left(q_{3}, q_{4}\right)$ is lower bounded and $\dot{V}\left(q_{3}, q_{4}\right)$ is negative semi-definite, this implies that $\dot{V}\left(q_{3}, q_{4}\right) \leq V(0,0)$ and, therefore, that $q_{3}$ and $q_{4}$ are bounded.

To use Barbalat's lemma, let us check the uniform continuity of $\dot{V}\left(q_{3}, q_{4}\right)$. The derivative of $\dot{V}\left(q_{3}, q_{4}\right)$ is

$$
\begin{aligned}
\ddot{V}= & -2 \frac{\sigma_{02}}{m_{2}} q_{4}\left(-\frac{\sigma_{02}}{m_{2}} q_{4}-\frac{k}{m_{2}} q_{3}+\frac{k}{2 m_{2}}\left(\left|\delta q-\frac{c}{2}\right|\right.\right. \\
& \left.\left.+\left|x_{1 d}+\frac{c}{2}\right|-\left|\delta q+\frac{c}{2}\right|-\left|x_{1 d}-\frac{c}{2}\right|\right)\right) .
\end{aligned}
$$

This shows that $\ddot{V}$ is bounded, since $q_{3}$ and $q_{4}$ were shown above to be bounded. Hence, $\dot{V}$ is uniformly continuous.
Application of Barbalat's lemma then indicates that $q_{4} \rightarrow$ 0 as $t \rightarrow \infty$.

Thus, coordinates $\left(q_{3}, q_{4}\right)$ converge to $(\epsilon, 0)$, where $\epsilon \in[-c / 2, c / 2]$, which means that an error will be almost always present on the position of the underactuated mass.

In what follows, the purpose is to reduce this position error in the unactuated mass by the application of $H_{\infty}$ control design to the previous system. Moreover, the perturbation $w_{2}$ and the Coulomb friction $F_{c 2} \operatorname{sign}\left(q_{4}\right)$, as well as their effects on the output $q_{3}$ of the closed-loop system, are intended to be reduced through $H_{\infty}$ control. Thus, the regulation control problem for $q_{3}$ in the system (23) can be expressed formally as an $H_{\infty}$ control problem.

\section{4. $H_{\infty}$ control design}

In the sequel, the investigation will be confined to the $H_{\infty}$ position regulation problem for system (23), where

(i) the output to be controlled is given by

$$
z=\rho\left[\begin{array}{c}
0 \\
q_{3}
\end{array}\right]+\left[\begin{array}{l}
1 \\
0
\end{array}\right] u_{\infty}
$$

with a positive weight coefficient $\rho$;

(ii) the positions $q_{1}, q_{3}$ are the available measurements

$$
y=\left[\begin{array}{l}
q_{1}+x_{d 1} \\
q_{3}+x_{d 2}
\end{array}\right] .
$$

The $H_{\infty}$ control problem in question is thus stated as follows: Given the system representation (23), (30), (31) and a real number $\gamma>0$, it is required to find (if any) a causal dynamic feedback controller

$$
u_{\infty}=K(q),
$$

with internal state $q \in \mathbb{R}^{4}$ such that the undisturbed closed-loop state $q_{3}$ is locally uniformly asymptotically stable around the origin and its $L_{2}$ gain is locally less than $\gamma$.

Now, $H_{\infty}$ control design for the system (23) will be presented, which is modeled by the equations presented by Aguilar et al. (2003) or Isidori and Astolfi (1992):

$$
\begin{aligned}
& \dot{q}=\Gamma(q)+\Phi(q)+g_{1}(q) w+g_{2}(q) u_{\infty} \\
& z=h_{1}(q)+k_{12}(q) u_{\infty} \\
& y=h_{2}(q)+k_{21}(q) w
\end{aligned}
$$


where

$$
\begin{aligned}
& \Gamma(q)=\left[\begin{array}{c}
q_{2} \\
-q_{1}-q_{2} \\
q_{4} \\
-\frac{\sigma_{02}}{m_{2}} q_{4}+\frac{k}{m_{2}} q_{1}-\frac{k}{m_{2}} q_{3}
\end{array}\right], \\
& \Phi(q)=\left[\begin{array}{c}
0 \\
0 \\
0 \\
-\frac{F_{c 2}}{m_{2}} \operatorname{sign}\left(q_{4}\right)+\frac{k}{2 m_{2}}\left(\left|x_{1 d}+\frac{c}{2}\right|-\right. \\
\left.\left|x_{1 d}-\frac{c}{2}\right|+\left|\delta q-\frac{c}{2}\right|-\left|\delta q+\frac{c}{2}\right|\right)
\end{array}\right], \\
& g_{1}(q)=\left[\begin{array}{llll}
0 & 0 & 0 & 0 \\
0 & 0 & 0 & 0 \\
0 & 0 & 0 & 0 \\
0 & 1 & 0 & 0
\end{array}\right], \quad g_{2}(q)=\left[\begin{array}{l}
0 \\
1 \\
0 \\
0
\end{array}\right] \text {, } \\
& h_{1}(q)=\rho\left[\begin{array}{c}
0 \\
q_{3}
\end{array}\right], \quad h_{2}(q)=\left[\begin{array}{l}
q_{1}+x_{1_{d}} \\
q_{3}+x_{2_{d}}
\end{array}\right] \text {, } \\
& k_{12}(q)=\left[\begin{array}{l}
1 \\
0
\end{array}\right], \quad k_{21}(q)=\left[\begin{array}{llll}
0 & 0 & 1 & 0 \\
0 & 0 & 0 & 1
\end{array}\right] \text {. }
\end{aligned}
$$

From the system (23), let us derive a local solution to the $H_{\infty}$ regulation problem. Therefore, the local solution to the $H_{\infty}$-position regulation problem subject to $36-(38)$ has the following structure (see Aguilar et al., 2003; Doyle et al., 1989; Kwakernaak, 1993):

$$
\begin{aligned}
& \dot{x}=A x+B_{1} w+B_{2} u, \\
& z=C_{1} x+D_{12} u, \\
& y=C_{2} x+D_{21} w,
\end{aligned}
$$

where

$$
\begin{aligned}
A & =\frac{\partial \Gamma}{\partial x}(0), & B_{1}=g_{1}(0), & B_{2}=g_{2}(0), \\
C_{1} & =\frac{\partial h_{1}}{\partial x}(0), & D_{12}=k_{12}(0), & C_{2}=\frac{\partial h_{2}}{\partial x}(0), \\
D_{21} & =k_{21}(0), & &
\end{aligned}
$$

with matrices $A, B_{1}, B_{2}, C_{1}, C_{2}, D_{12}, D_{21}$ of appropriate dimensions. The general state-space representation with nonzero feedthrough terms $D_{11}$ and $D_{22}$ can be treated as in the work of Safonov et al. (1989) by constructing an equivalent problem with $D_{11}=0$ and $D_{22}=0$. In addition, the simplifying assumptions

$\left(A_{1}\right)\left(A, B_{1}\right)$ is stabilizable and $\left(C_{1}, A\right)$ is detectable,

$\left(A_{2}\right)\left(A, B_{2}\right)$ is stabilizable and $\left(C_{2}, A\right)$ is detectable,

$\left(A_{3}\right) D_{12}^{T} C_{1}=0$ and $D_{12}^{T} D_{12}=I$,

$\left(A_{4}\right) B_{1} D_{21}^{T}=0$ y $D_{21} D_{21}^{T}=I$,

presented by Doyle et al. (1989), are made throughout.
A local solution is then derived by means of the perturbed Riccati equation (Aguilar et al., 2003)

$$
\begin{aligned}
0= & P_{\varepsilon} A+A^{T} P_{\varepsilon}+C_{1}^{T} C_{1} \\
& +P_{\varepsilon}\left[\frac{1}{\gamma^{2}} B_{1} B_{1}^{T}-B_{2} B_{2}^{T}\right] P_{\varepsilon}+\varepsilon I .
\end{aligned}
$$

There exists a positive constant $\varepsilon_{0}$ such that the system of the perturbed algebraic Riccati equation has a unique positive definite symmetric solution $P_{\varepsilon}$ for each $\varepsilon \in$ $\left(0, \varepsilon_{0}\right)$. Equation (41) is utilised to derive a local solution to the $H_{\infty}$-control problem for a mechanical system with friction and backlash (36)- 38 .

Let $P_{\varepsilon}$ be a positive definite solution to (41) under some $\varepsilon>0$. Then the output feedback is given by

$$
u_{\infty}=-g_{2}^{T}(q) P_{\varepsilon} q
$$

which is a local solution of the $H_{\infty}$-control problem. The $H_{\infty}$ control stage has two purposes: to achieve closed-loop stability and to attenuate the influence of the external input $w$ on the penalty variable $z$. Perturbation attenuation depends on the specific class of external signals to be considered and/or the performance criteria chosen to evaluate the penalty variable. Given a real number $\gamma>0$, it is said that the system (33), (42) has $\mathcal{L}_{2}$ gain less than $\gamma$ if the response $z$, resulting from $w$ for initial state $q(0)=0$, satisfies

$$
\int_{0}^{T} z^{T}(t) z(t) \mathrm{d} t \leq \gamma^{2} \int_{0}^{T} w^{T}(t) w(t) \mathrm{d} t
$$

for all $T>0$ and all piecewise continuous functions $w(t)$, for which the corresponding state trajectory of the closed-loop system, initialised at the origin, remains in some neighbourhood of this point.

\section{Finite time velocity observer design}

This section explains a finite time velocity observer for system 111. The following observer was taken from the previous work of Orlov et al. (2011). The system (11) has the output $y=\left[q_{1}, q_{3}\right]^{T}$ and it is assumed that the variables $q_{2}$ and $q_{4}$ are not available.

The finite time observer considered has the form

$$
\begin{aligned}
\dot{\hat{q}}_{1}= & \hat{q}_{2}+k_{1}\left|e_{1}\right|^{\frac{1}{2}} \operatorname{sign}\left(e_{1}\right)+k_{2} e_{1}, \\
\dot{\hat{q}}_{2}= & \frac{1}{m_{1}}\left[-\sigma_{01} \hat{q}_{2}-f\left(\hat{q}_{1}+x_{1_{d}}, \hat{q}_{3}+x_{2_{d}}\right)+u\right] \\
& +k_{3} \operatorname{sign}\left(e_{1}\right)+k_{4} e_{1}, \\
\dot{\hat{q}}_{3}= & \hat{q}_{4}+k_{5}\left|e_{3}\right|^{\frac{1}{2}} \operatorname{sign}\left(e_{3}\right)+k_{6} e_{3}, \\
\dot{\hat{q}}_{4}= & \frac{1}{m_{2}}\left[-\sigma_{02} \hat{q}_{4}+f\left(\hat{q}_{1}+x_{1_{d}}, \hat{q}_{3}+x_{2_{d}}\right)\right] \\
& +k_{7} \operatorname{sign}\left(e_{3}\right)+k_{8} e_{3} .
\end{aligned}
$$


The variables $e_{i}$ with $i=1, \ldots 4$ stand for the observation errors, which are given by $e_{i}=q_{i}-\hat{q}_{i}$. The gains of the observer are given by $k_{i}$. As (44) possess discontinuous right-hand sides, the dynamics of such equations are defined throughout in the sense of Filippov (1988). The dynamics of the observation errors considering $\mp f\left(q_{1}, q_{3}\right) \pm f\left(\hat{q}_{1}, \hat{q}_{3}\right)=\mp k e_{1} \pm k e_{3}$ are given by

$$
\begin{aligned}
\dot{e}_{1}= & e_{2}-k_{1}\left|e_{1}\right|^{\frac{1}{2}} \operatorname{sign}\left(e_{1}\right)-k_{2} e_{1}, \\
\dot{e}_{2}= & \frac{1}{m_{1}}\left[-\sigma_{01} e_{2}-k e_{1}+k e_{3}\right] \\
& -k_{3} \operatorname{sign}\left(e_{1}\right)-k_{4} e_{1}, \\
\dot{e}_{3}= & e_{4}-k_{5}\left|e_{3}\right|^{\frac{1}{2}} \operatorname{sign}\left(e_{3}\right)-k_{6} e_{3}, \\
\dot{e}_{4}= & \frac{1}{m_{2}}\left[-\sigma_{02} e_{4}+k e_{1}-k e_{3}\right] \\
& -k_{7} \operatorname{sign}\left(e_{3}\right)-k_{8} e_{3} .
\end{aligned}
$$

For stability purposes, according to Orlov et al. (2011), the term

$$
\left|\frac{1}{m_{j}}\left[-\sigma_{0 j} e_{(2 \times j)}+k e_{\left(\frac{4}{j}-1\right)}\right]\right| \leq \Psi_{j},
$$

with $j=1,2$ and $\Psi_{j}>0$ must meet the conditions

$$
\begin{aligned}
& \Psi_{1}<\min \left\{\frac{k_{1}}{2}, \frac{k_{1} k_{3}}{1+k_{1}}\right\}, \\
& \Psi_{2}<\min \left\{\frac{k_{5}}{2}, \frac{k_{5} k_{7}}{1+k_{5}}\right\} .
\end{aligned}
$$

Then the system 45 is globally finite time stable whenever the inequalities (47) are satisfied; the corresponding settling-time functions are given by Orlov et al. (2011). The block diagram representation of the closed-loop system can be seen in Fig. 3 .

For stability purposes of the overall closed-loop system, the observer will be initialized before the

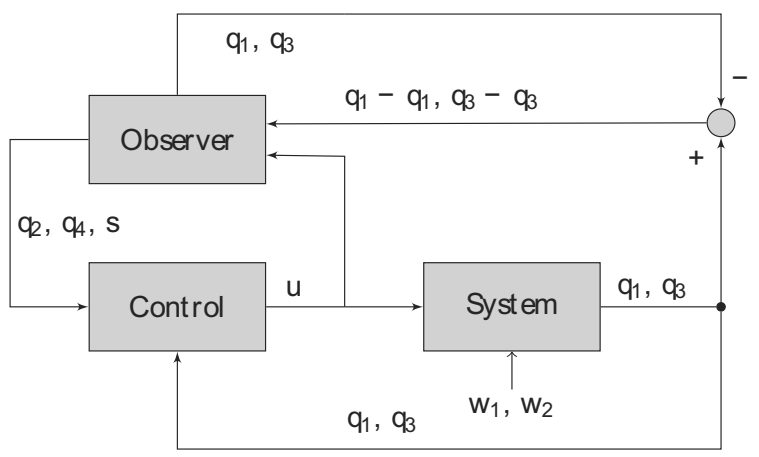

Fig. 3. Block diagram of the closed-loop system. implementation of the controller (14)-15]; this means that the controller will be activated after the settling-time of the observer has elapsed, when $\hat{q}_{2}=q_{2}$ and $\hat{q}_{4}=q_{4}$.

\section{Experimental results}

Performance issues and robustness properties of the proposed controller (14)-16 using the disturbance attenuator $u_{\infty}$ in (42) were tested on the ECP-210 platform, made by the Educational Control Products company. This platform was modified to have a backlash as in Fig. 4 Since the only state measurements available are $x_{1}$ and $x_{2}$, the proposed discontinuous velocity observer (44) is applied to have access to the remaining states.

An Intel ${ }^{\circledR}$ Core 2 Quad computer was used, and the Simulink ${ }^{\circledR}$ software was employed as an interface; the sample rate for the data acquisition was set at $0.001 \mathrm{~s}$. The platform was configured to have the dynamical behavior as the mechanical system depicted in Fig. 1. Particularly, the nonlinear characteristic of the backlash shown in Fig. 2 is taken into account and reproduced by adding a modification to the mechanical system; see Fig. 4. The modification to the system consists of a double end stud screw, which is linked by a spring to the first mass, leaving a backlash between the spring and the second mass. Thus, rebounds may occur between the actuated and unactuated masses.

The parameters of the mechanical system were considered as in Table 1. The desired position of the underactuated mass is given by $x_{2 d}=2.0 \mathrm{~cm}$. The controller feedback gains were $\beta=0.35 N, \lambda=0.05$, $\delta_{0}=0.1, \alpha=10$ and $p=10$. It is worth mentioning that friction terms were calculated in accordance with the methodologies presented by Virgin (2000).

Table 1. Nominal parameters.

\begin{tabular}{|l|l|r|}
\hline Description & Notation & Value \\
\hline \hline First mass & $m_{1}$ & $1 \mathrm{~kg}$ \\
Second mass & $m_{2}$ & $0.6 \mathrm{~kg}$ \\
Clearance & $\mathrm{c}$ & $1 \mathrm{~cm}$ \\
Viscous friction & $\sigma_{01}$ & $10 \mathrm{~kg} / \mathrm{s}$ \\
Viscous friction & $\sigma_{02}$ & $17 \mathrm{~kg} / \mathrm{s}$ \\
Coulomb friction & $F_{c 1}$ & $0.1 \mathrm{~N}$ \\
Coulomb friction & $F_{c 2}$ & $0.1 \mathrm{~N}$ \\
Spring stiffness & $k$ & $375 \mathrm{~N} / \mathrm{m}$ \\
\hline
\end{tabular}

Additionally, an external but bounded force perturbation governed by

$$
w_{1}=0.03 \sin (t) N
$$

is applied. For the selected $\gamma=1, \rho=1$ and $\varepsilon=0$, the corresponding perturbed Riccati equation (41) has a positive definite solution 


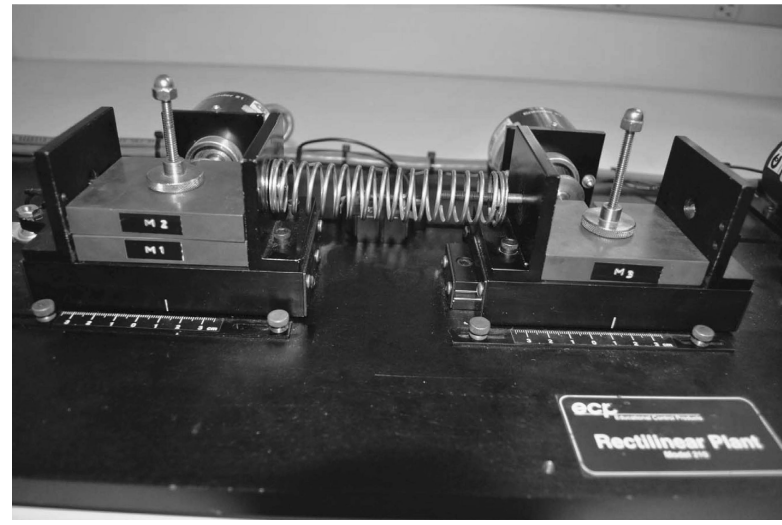

Fig. 4. Experimental platform ECP-210 configured to have gear play.

$P_{\epsilon}=\left[\begin{array}{cccc}23.3243 & 1.4093 & -18.7980 & 0.0030 \\ 1.4093 & 1.1951 & -0.5769 & -0.0304 \\ -18.7980 & -0.5769 & 17.3192 & 0.0006 \\ 0.0030 & -0.0304 & 0.0006 & 0.0300\end{array}\right]$,

which has been numerically found using MATLAB. It is noteworthy that the gain parameters of the controller (15) were tuned according to some characteristics such as the velocity of response, robustness and control signal amplitude. These characteristics were taken into account first when performing numerical simulations. Based on the values of the gain parameters obtained by numerical simulations, the experimental gain parameters were tuned by trial and error; therefore, it is possible that other values of the controller gain parameters yield better results. The perturbation $w_{2}$ is considered inherent to the mechanical system.

On the other hand, the finite time velocity observer gains were set to $k_{1}=k_{3}=k_{5}=k_{7}=1, k_{2}=k_{6}=7$, and $k_{4}=k_{8}=10$. The values for the initial conditions were $q_{1}(0)=0 \mathrm{~m}, q_{2}(0)=0 \mathrm{~m} / \mathrm{s}, q_{3}(0)=0 \mathrm{~m}, q_{4}(0)=$ $0 \mathrm{~m} / \mathrm{s}$ and $s(0)=0$. The observer's initial conditions were set as $\hat{q}(0)=0 \in \mathbb{R}^{4}$.

6.1. $H_{\infty}$ controller comparison. In order to appreciate the performance of the sliding mode $H_{\infty}$ controller, a comparison is made against an $H_{\infty}$ control scheme presented by Rascón et al. (2016); also, a nonvanishing perturbation was considered as in (48). The gains of the $H_{\infty}$ controller were set as in the work of Rascón et al. (2016) the velocities of the system were estimated using a filter implemented therein. The results can be seen in Figs. 5, 8 , where the sliding mode $H_{\infty}$ controller yields better performance than the $H_{\infty}$ controller, with a smaller settling time and smaller position errors using the proposed control approach, which are more evident in the perturbed case.

In Fig. 5 it can be seen that the position errors $q_{1}$ and $q_{3}$, corresponding to the actuated and underactuated mass, respectively, tend to the reference in less than 1.5 seconds. The observed velocities of the masses $\hat{q}_{2}$ and $\hat{q}_{4}$ corresponding to the first and second masses were obtained from the observer (44), and they were used as feedback in the controller.

In Fig. 6, where the system at hand is affected by a nonvanishing perturbation (except for nonlinear phenomena, such as discontinuous friction and backlash), the position errors $q_{1}$ and $q_{3}$ tend to their nominal values in less than 1.5 seconds. Since the bound of the perturbation satisfies $M<\beta / N(s)$, the system is insensitive to that perturbation. On the other hand, the $H_{\infty}$ control scheme only diminishes the effect of the perturbations on the outputs $q_{1}$ and $q_{3}$. In Figs. 7 and 8 , we have the control signals, the sliding motions $s$, and phase portraits of the unperturbed and perturbed cases, respectively. In the control signal of the proposed approach, high frequencies can be seen due to the discontinuous term used in controller design, although the control signal of the $H_{\infty}$ implementation has a bigger transient than the one of the proposed approach; note that the $H_{\infty}$ algorithm is not insensitive to external perturbations. Also, in Figs. 7 and 8 we show the sliding motion variable $s$. For both experiments the plots are very similar due to the insensitivity of the algorithm to certain bounded perturbations. Finally in the same figures the phase portraits of both links are shown.

6.2. Test under parametric variation. Now, a test is made under a parameter variation. Let us change the spring of $375 \mathrm{~N} / \mathrm{m}$ for a spring of $105 \mathrm{~N} / \mathrm{m}$. This change affects directly the closed-loop performance since the proposed control law (15) uses the spring constant in order to compensate the contact force between both masses; note that the spring is changed on the physical system. In the control law (15) the value of the spring is kept as $375 \mathrm{~N} / \mathrm{m}$. The results can be seen in Figs. 9 and 10. where the sliding mode- $H_{\infty}$ controller yields an acceptable performance despite the controller not being originally designed to absorb a spring variation.

\section{Conclusions}

We developed a fully practical framework for sliding mode control involving an $H_{\infty}$ control methodology in sliding surface design. The aforementioned design procedure was shown to be eminently suited to solving a position regulation problem for a mechanical system with friction, backlash, and external perturbation. The position error of the underactuated mass is reduced by the $H_{\infty}$ control stage. The proposed sliding mode $H_{\infty}$ 

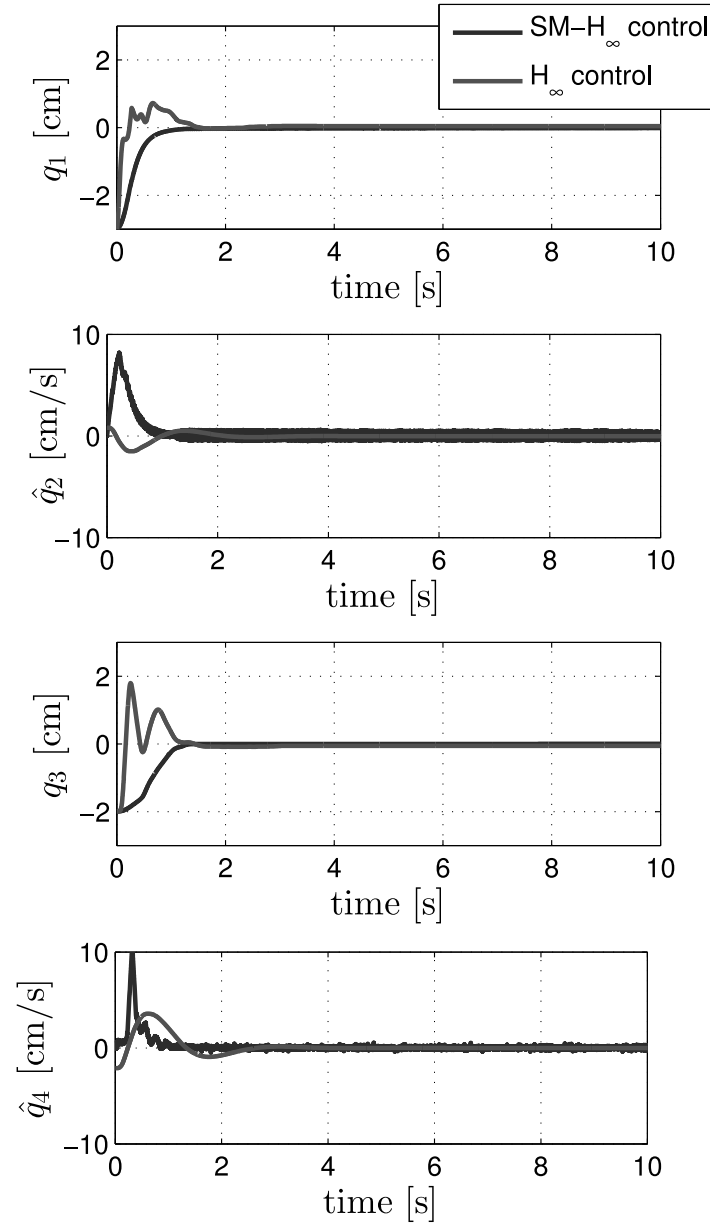

Fig. 5. Experimental results using the sliding mode $H_{\infty}$ controller (dark gray) and an $H_{\infty}$ controller (light gray). For the implementation of the sliding mode $H_{\infty}$ controller only mass position measurements were available; the velocities $\hat{q}_{2}$ and $\hat{q}_{4}$ were obtained using an observer.

output regulation synthesis is suited to absorb bounded perturbations $\sup _{t}\left|w_{1}(t)\right| \leq M$ and Coulomb friction $F_{c 1} \operatorname{sign}\left(q_{2}\right)$ on the actuated mass that satisfied $\beta / N(s)-$ $M / m_{1}-F_{c 1} / m_{1}>0$. Whenever this inequality is not satisfied, the controller will attenuate the perturbations and dead zone model discrepancies, but it is enough to increase the gain parameter $\beta$ in order to fulfill the stability inequality. Moreover, the $H_{\infty}$ control stage will attenuate perturbations $w_{2}(t)$ affecting the underactuated mass. In the unperturbed case, the position error of the underactuated mass will be inside the set $[-c / 2, c / 2]$, and this error will be attenuated by the $H_{\infty}$ control stage. The main contribution of this work is the design of a sliding mode $H_{\infty}$ controller to regulate the position in an underactuated mechanical system with backlash, Coulomb friction in both masses, and affected by matched and unmatched perturbations. The effectiveness of
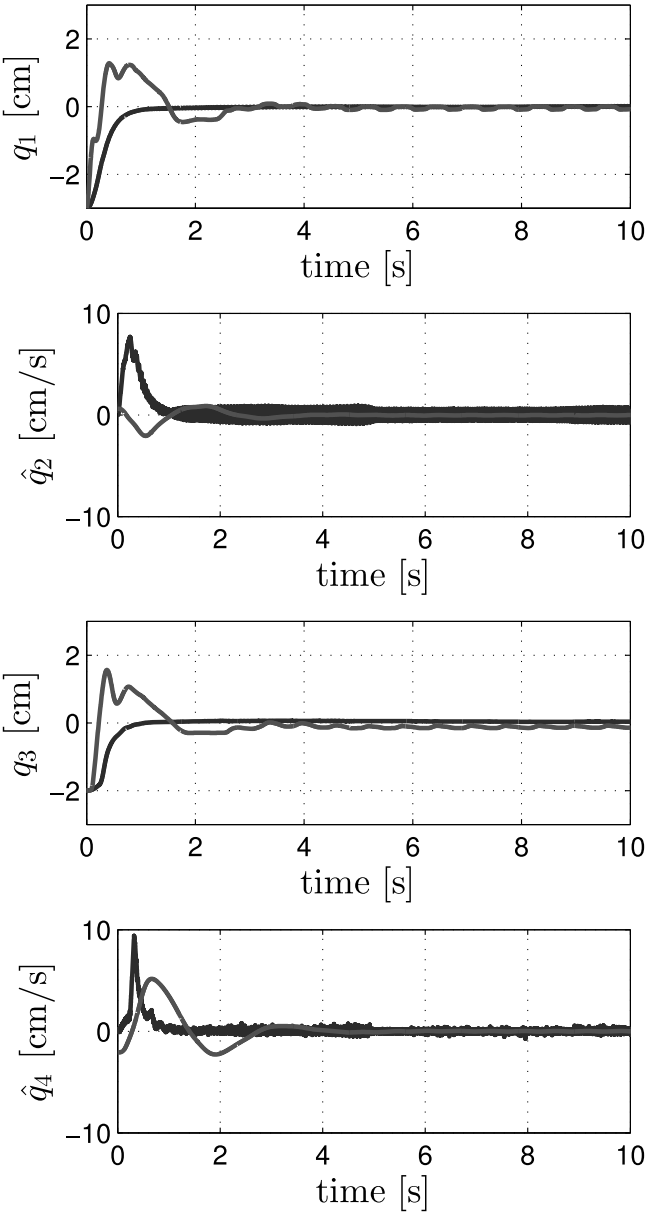

Fig. 6. Experimental results using the sliding mode $H_{\infty}$ controller (dark gray) and an $H_{\infty}$ controller (light gray), where mass position measurements were used for both controllers: a perturbed case.

the design procedure was supported by experiments on the platform ECP-210 modified to have backlash and parameter variation. One possible application of this class of system could be the modeling of a car suspension or cooperative systems.

\section{References}

Adetola, V., DeHaan, D. and Guay, M. (2009). Adaptive model predictive control for constrained nonlinear systems, Systems \& Control Letters 58(5): 320-326.

Aguilar, L., Orlov, Y. and Acho, L. (2003). Nonlinear $\mathcal{H}_{\infty}$ control of nonsmooth time varying systems with application to friction mechanical manipulators, Automatica 39(9): 1531-1542.

Brahim, A.B., Dhahri, S., Hmida, F.B. and Sellami, A. (2015). An $\mathcal{H}_{\infty}$ sliding mode observer for Takagi-Sugeno nonlinear systems with simultaneous actuator and sensor faults, International Journal of Applied Mathemat- 

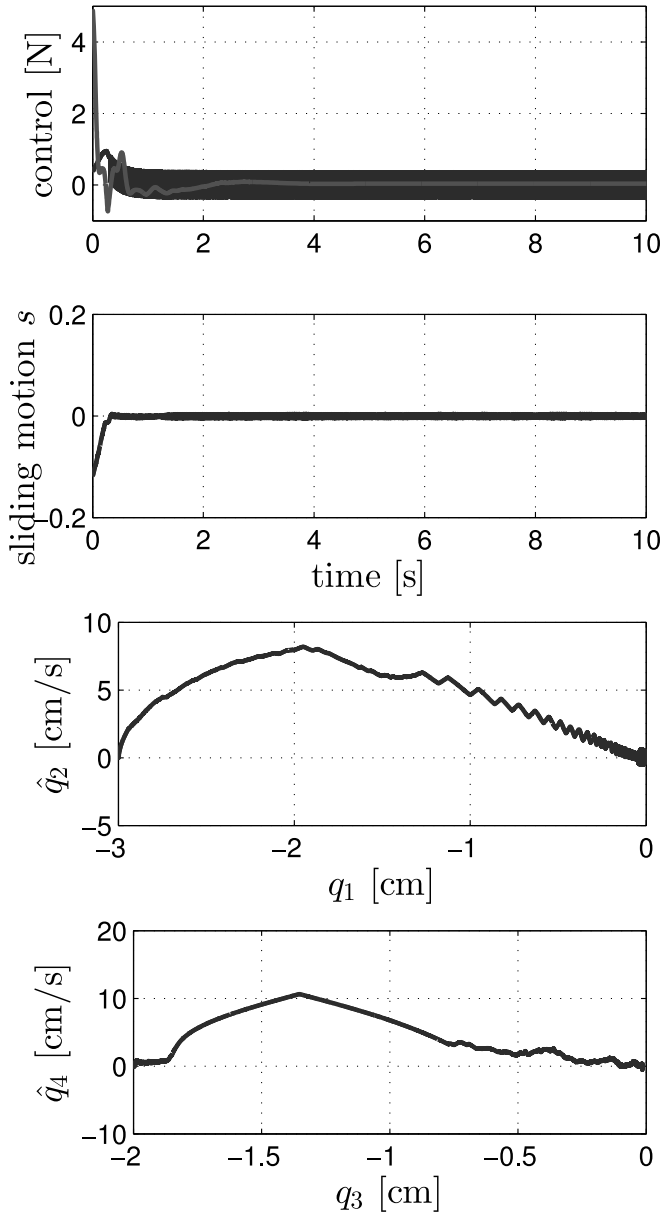

Fig. 7. Experimental results showing the control signal, sliding motion $s$, and phase portrait of both links. The $H_{\infty}$ control algorithm does not include a sliding surface in its design.

ics and Computer Science 25(3): 547-559, DOI: 10.1515/amcs-2015-0041.

Branicky, M. (1998). Multiple Lyapunov functions and other analysis tools for switched and hybrid systems, IEEE Transactions on Automatic Control 43(4): 475-482.

Brogliato, B. (1999). Nonsmooth Mechanics, Springer, London.

Brogliato, B., Niculescu, S.-I. and Orhant, P. (1997). On the control of finite-dimensional mechanical systems with unilateral constraints, IEEE Transactions on Automatic Control 42(2): 200-215.

Castaños, F. and Fridman, L. (2006). Analysis and design of integral sliding manifolds for systems with unmatched perturbations, IEEE Transactions on Automatic Control 51(5): 853 - 858 .

Castaños, F. and Fridman, L. (2011). Dynamic switching surfaces for output sliding mode control: An $H_{\infty}$ approach, Automatica 47(9): 1957-1961.

Chang, Y.-C. and Lee, C.-H. (1999). Robust tracking control for constrained robots actuated by DC motors without velocity
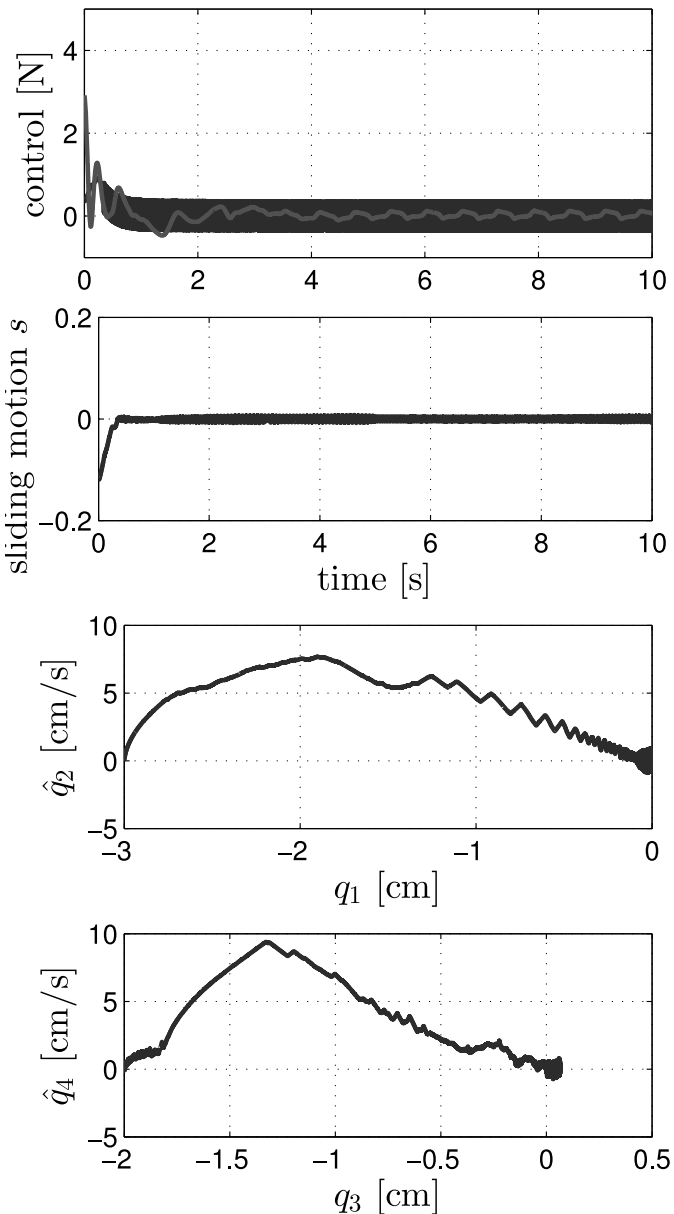

Fig. 8. Results showing the control signal, sliding motion $s$, and phase portraits of both links using the sliding mode $H_{\infty}$ controller (dark gray) and an $H_{\infty}$ controller (light gray): a perturbed case.

measurements, IEE Proceedings-Control Theory and Applications 146(2): 147-156.

Chiu, C.-S., Lian, K.-Y. and Wu, T.-C. (2004). Robust adaptive motion/force tracking control design for uncertain constrained robot manipulators, Automatica 40(12): 2111-2119.

Christophersen, F.J. (2007). Optimal Control of Constrained Piecewise Affine Systems, Lecture Notes in Control and Information Sciences, Springer, Berlin/Heidelberg.

Doyle, J.C., Glover, K., Khargonekar, P.P. and Francis, B.A. (1989). State-space solutions to standard $\mathcal{H}_{2}$ and $\mathcal{H}_{\infty}$ control problems, IEEE Transactions on Automatic Control 34(8): 831-847.

Durmaz, B., Özgören, M. K. and Salamci, M. U. (2011). Sliding mode control for non-linear systems with adaptive sliding surfaces, Transactions of the Institute of Measurement and Control 34(1): 56-90.

Fallaha, C.J., Saad, M., Kanaan, H.Y. and Al-Haddad, K. (2011). Sliding-mode robot control with exponential 

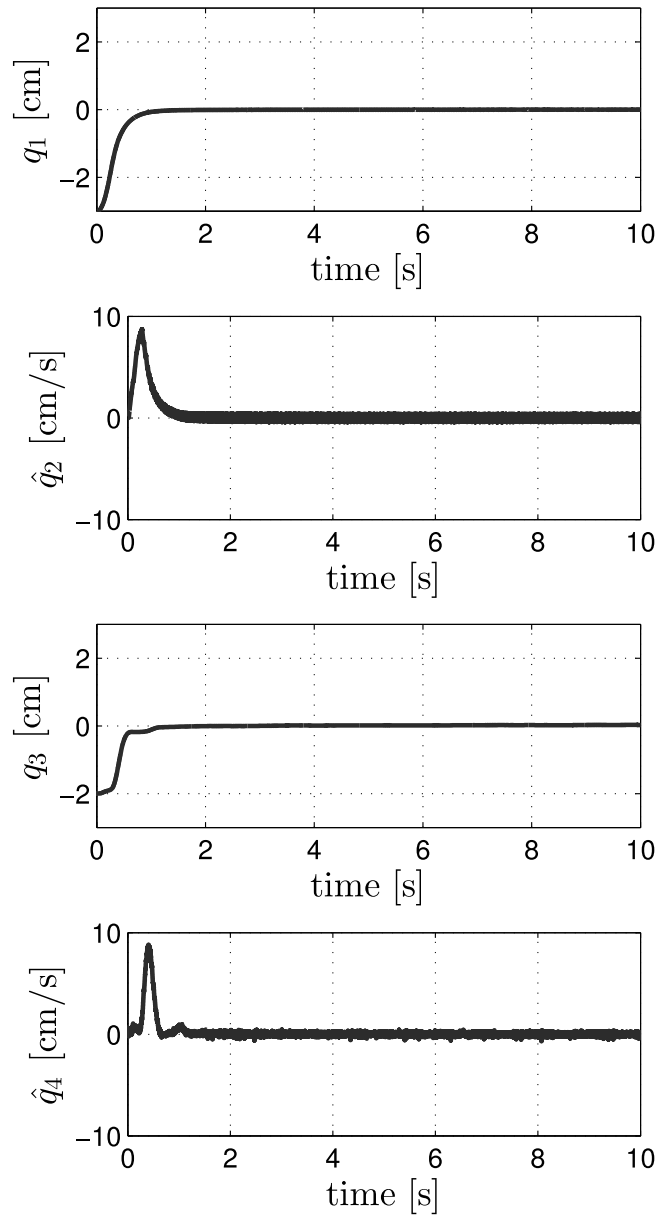

Fig. 9. Performance of the closed-loop system under spring value variation.

reaching law, IEEE Transactions on Industrial Electronics 58(2): 600-610.

Filippov, A.F. (1988). Differential Equations with Discontinuous Right-Handsides, Kluwer, Dordercht.

Ghafari-Kashani, A., Faiz, J. and Yazdanpanah, M. (2010). Integration of non-linear $\mathcal{H}_{\infty}$ and sliding mode control techniques for motion control of a permanent magnet synchronous motor, IET Electric Power Applications 4(4): 267-280.

Isidori, A. (2000). A tool for semiglobal stabilization of uncertain non minimum-phase nonlinear systems via output feedback, IEEE Transactions on Automatic Control 48(10): 1817-1827.

Isidori, A. and Astolfi, A. (1992). Disturbance attenuation and $\mathcal{H}_{\infty}$-control via measurement feedback in nonlinear systems, IEEE Transactions on Automatic Control 37(9): 1283-1293.

Kazerooni, H. (1990). Contact instability of the direct drive robot when constrained by a rigid environment, IEEE Transactions on Automatic Control 35(6): 710-714.
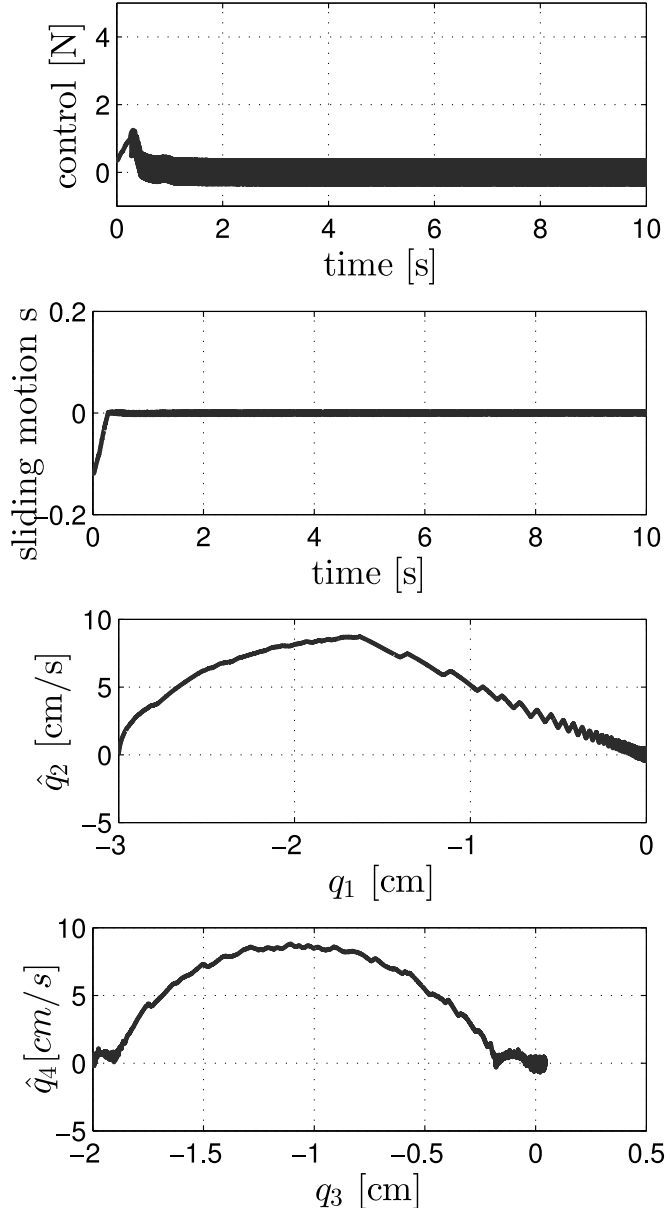

Fig. 10. Results showing the control signal, sliding motion $s$, and phase portraits of both links when the closed-loop system is under spring value variation.

Khalil, H. (2002). Nonlinear Systems, Prentice Hall, Upper Saddle River, NJ.

Kwakernaak, H. (1993). Robust control and $\mathcal{H}_{\infty}$-optimization-tutorial paper, Automatica 29(2): 255-273.

Leine, R. I. and Van de Wouw, N. (2010). Stability and Convergence of Mechanical Systems with Unilateral Constraints, Springer, Berlin.

Lian, J. and Zhao, J. (2010). Robust $H$-infinity integral sliding mode control for a class of uncertain switched nonlinear systems, Journal of Control Theory and Applications 8(4): 521-526.

Lian, K.-Y. and Lin, C.-R. (1998). Sliding-mode motion/force control of constrained robots, IEEE Transactions on Automatic Control 43(8): 1101-1103.

Luo, N., Tan, Y. and Dong, R. (2015). Observability and controllability analysis for sandwich systems with backlash, International Journal of Applied Mathematics and Computer Science 25(4): 803-814, DOI: 10.1515/amcs-2015-0057. 
Mansard, N. and Khatib, O. (2008). Continuous control law from unilateral constraint, IEEE International Conference on Robotics and Automation, ICRA 2008, Pasadena, CA, USA, pp. 3359-3364.

Menini, L. and Tornambe, A. (2001). Dynamic position feedback stabilisation of multidegrees-of-freedom linear mechanical systems subject to nonsmooth impacts, IEE Proceedings-Control Theory and Applications 148(6): 488-496.

Orlov, Y., Aoustin, Y. and Chevallereau, C. (2011). Finite time stabilization of a perturbed double integrator. I: Continuous sliding mode-based output feedback synthesis, IEEE Transactions on Automatic Control 56(3): 614-618.

Paden, B. and Sastry, S. (1987). A calculus for computing Filippov's differential inclusion with application to the variable structure control of robot manipulators, IEEE Transactions on Circuits and Systems 34(1): 73-81.

Perez, M., Jimenez, E. and Camacho, E. (2010). Design of an explicit constrained predictive sliding mode controller, IET Control Theory \& Applications 4(4): 552 -562.

Potini, A., Tornambe, A., Menini, L., Abdallah, C. and Dorato, P. (2006). Finite-time control of linear mechanical systems subject to non-smooth impacts, 14th Mediterranean Conference on Control and Automation, MED'06, Ancona, Italy, pp. 1-5.

Rascón, R., Alvarez, J. and Aguilar, L. (2012). Sliding mode control with $\mathcal{H}_{\infty}$ : Attenuator for unmatched disturbances in a mechanical system with friction and a force constraint, 12th International Workshop on Variable Structure Systems (VSS), Mumbai, Maharashtra, India, pp. 434-439.

Rascón, R., Álvarez, J. and Aguilar, L.T. (2014). Control robusto de posición para un sistema mecánico subactuado con fricción y holgura elástica, Revista Iberoamericana de Automática e Informática Industrial 11(3): 275-284.

Rascón, R., Alvarez, J. and Aguilar, L.T. (2016). Discontinuous $\mathcal{H}_{\infty}$ control of underactuated mechanical systems with friction and backlash, International Journal of Control, Automation and Systems 14(5): 1213-1222.

Sabanovic, A., Elitas, M. and Ohnishi, K. (2008). Sliding modes in constrained systems control, IEEE Transactions on Industrial Electronics 55(9): 3332-3339.

Safonov, M.G., Limebeer, D.J.N. and Chiang, R.Y. (1989). Simplifying the $\mathcal{H}_{\infty}$ theory via loop-shifting, matrix-pencil and descriptor concepts, International Journal of Control 50(6): 2467-2488.

Shevitz, D. and Paden, B. (1994). Lyapunov stability theory of nonsmooth systems, IEEE Transactions on Automatic Control 39(9): 1910-1914.

Tseng, C.-S. (2005). Mixed $\mathcal{H}_{2} / \mathcal{H}_{\infty}$ adaptive tracking control design for uncertain constrained robots, Asian Journal of Control 7(3): 296-309.

Utkin, V. (1978). Sliding Modes and Their Applications, Mir, Moscow.

Utkin, V. (1992). Sliding Modes in Control Optimization, Springer, Berlin.
Virgin, L.N. (2000). Introduction to Experimental Nonlinear Dynamics: A Case Study in Mechanical Vibration, Cambridge University Press, New York, NY.

Zhu, J. and Khayati, K. (2015). A new approach for adaptive sliding mode control: Integral/exponential gain law, Transactions of the Institute of Measurement and Control 38(4): 385-394.

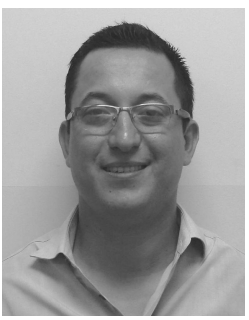

Raúl Rascón received his $\mathrm{PhD}$ degree in electronics and telecommunications from the Scientific Research and Higher Education Center of Ensenada, CICESE, Baja California, México, in 2012. He is currently a full time professor and researcher at the Autonomous University of Baja California, UABC. His research interests include robust control, nonlinear control, observers and applications to mechanical systems.

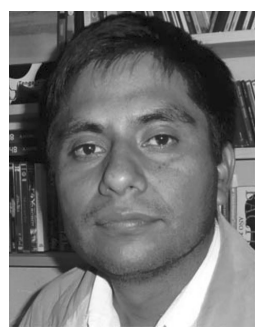

David Rosas was born in Tlaixpan, Mexico, in 1975. He received the BSc degree in electronics engineering from the Autonomous University of Puebla, Puebla, Mexico, in 1997, and the $\mathrm{MSc}$ and $\mathrm{PhD}$ degrees in electronics and telecommunications, with specialization in instrumentation and control, from the Scientific Research and Higher Education Center of Ensenada, Mexico, in 1999 and 2005, respectively. Since 1999, he has been a full professor with the Engineering Faculty, Autonomous University of Baja California, Mexicali, Mexico. His research interests are in the fields of nonlinear control, discontinuous systems, and synchronization.

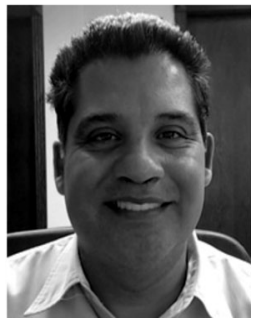

Daniel Hernández Balbuena was born in 1971. $\mathrm{He}$ received the BSc degree from Puebla Autonomous University, Puebla, Mexico, in 1996 and the MSc degree from Ensenada Center for Scientific Research and Higher Education, Baja California, Mexico, in 1999. He received the $\mathrm{PhD}$ degree at Baja California Autonomous University in 2010. His research interests are in the areas of time and frequency metrology, design and characterization of microwave devices and systems, RF measurements, research applications of unmanned aerial vehicles and digital image processing.

Received: 28 October 2016 Revised: 30 April 2017 Re-revised: 14 June 2017 Accepted: 28 June 2017 